\title{
Tight Riesz orders via congruences
}

\section{Elizabeth A.S. Loci}

The main theme of this thesis is to find, on the positive set of a lattice-ordered group, a lattice congruence such that the quotient lattice has 'geometrically' more easily recognizable elements - and to find a correspondence between compatible tight Riesz orders and subsets of the quotient lattice.

We consider the topological interior of a topological lattice-ordered group $G$. We show that it can determine a compatible tight Riesz order and, moreover, that it is obtained by deleting prime subgroups from the positive set of $G$ - thus giving an alternate proof to a central result of Reilly [1, Theorem 2.6], and suggesting that compatible tight Riesz orders are natural in the context of topological lattice-ordered groups.

We define a particular type of compatible tight Riesz order on the group $C(X)$ of continuous real-valued functions on a topological space, namely $z$-full tight Riesz orders. We then characterize z-full tight Riesz orders (respectively, their maximal tangents) in terms of certain lattice-ideals (respectively, filters) of the zero-set lattice.

We extend Rellly's characterization [1] of compatible tight Riesz orders on divisible abelian lattice-ordered groups to divisible normal-valued lattice-ordered groups, and use this to establish when the set of weak units of such a group determines a compatible tight Riesz order. Given these conditions we set up a correspondence between compatible tight Riesz orders and certain filters of the carrier lattice.

We consider the group, $A(\Omega)$, of order-preserving permutations of a totally-ordered field. We define a particular type of compatible tight Riesz order, namely a $\sigma-f u l l$ tight Riesz order, and characterize them

Received 1 November 1977. Thesis submitted to La Trobe University, May 1977. Degree approved, October 1977. Supervisor: Dr G.E. Davis. 
(respectively, their maximal tangents) in terms of certain ideals (respectively, filters) of the fixed-point lattice.

\section{Reference}

[1] N.R. Reilly, "Compatible tight Riesz orders and prime subgroups", Glasgow Math. J. 14 (1973), 145-160. 